\title{
High-Power 1320-nm Wafer-Bonded VCSELs With Tunnel Junctions
}

\author{
V. Jayaraman, M. Mehta, A. W. Jackson, S. Wu, Y. Okuno, J. Piprek, Senior Member, IEEE, and \\ J. E. Bowers, Fellow, IEEE
}

\begin{abstract}
A new long-wavelength vertical-cavity surface-emitting laser structure is described that utilizes AlGaAs-GaAs mirrors bonded to AlInGaAs-InP quantum wells with an intracavity buried tunnel junction. This structure offers complete wavelength flexibility in the 1250-1650 $\mathrm{nm}$ fiber communication bands and reduces the high free-carrier losses and bonded junction voltage drops in previous devices. The intracavity contacts electrically bypass the bonded junctions to reduce threshold voltage. $\mathrm{N}$-type current spreading layers and undoped AlGaAs mirrors minimize optical losses. This has enabled $134{ }^{\circ} \mathrm{C}$ maximum continuous-wave lasing temperature, 2-mW room-temperature continuous-wave single-mode power, and $1-\mathrm{mW}$ single-mode power at $80^{\circ} \mathrm{C}$, in various devices in the $1310-1340 \mathrm{~nm}$ wavelength range.
\end{abstract}

Index Terms-Long wavelength, semiconductor lasers, tunnel diodes, vertical cavity, vertical-cavity surface-emitting laser (VCSEL), wafer bonding.

\section{INTRODUCTION}

$\mathbf{R}$ ECENT YEARS have seen a rich variety of approaches to long-wavelength vertical-cavity surface-emitting lasers (VCSELs) [1]-[9]. Both epitaxial mirror approaches and wafer-bonded mirror approaches have achieved room-temperature continuous-wave (RTCW) powers in the neighborhood of $1 \mathrm{~mW}$ or greater, and a few authors have reported continuous-wave $(\mathrm{CW})$ lasing beyond $100^{\circ} \mathrm{C}[1]-[4]$.

In the neighborhood of $1.3 \mu \mathrm{m}$, the best output power and temperature performance have been achieved by [1] using GaInNAs-GaAs active regions in conjunction with epitaxially grown GaAs-AlGaAs mirrors. References [2] and [3], which use an integrated 850-nm optical pump and wafer-bonded AlGaAs mirrors, have reported comparable room temperature single-mode powers at longer wavelengths than [1], but with reduced power relative to [1] at higher temperatures. Reference [1] reports RTCW single-mode powers of $2 \mathrm{~mW}, 1-\mathrm{mW} \mathrm{CW}$ single-mode power at $90^{\circ} \mathrm{C}$, and well above $110{ }^{\circ} \mathrm{C}$ max $\mathrm{CW}$ lasing temperature at wavelengths near $1267 \mathrm{~nm}$.

In the neighborhood of $1.55 \mu \mathrm{m}$, the best results to date have been achieved using the buried tunnel junction approach with

Manuscript received May 15, 2003. This work was supported by Walsin Lihwa and the UC SMART Program.

V. Jayaraman was with the Department of Electrical and Computer Engineering, University of California Santa Barbara, Santa Barbara, CA 93106-9560 USA. He is now with Praevium Research, Inc., Santa Barbara, CA 93111 USA (e-mail: praevium@aol.com)

M. Mehta, A. W. Jackson, S. Wu, Y. Okuno, J. Piprek, and J. E. Bowers are with the Department of Electrical and Computer Engineering, University of California Santa Barbara, Santa Barbara, CA 93106-9560 USA.

Digital Object Identifier 10.1109/LPT.2003.818652 one epitaxially grown AlInGaAs-InP mirror and one dielectric mirror [4], [5], [8].

Thus far, approaches based on epitaxially grown mirrors have suffered from wavelength restrictions within the $1250-1650 \mathrm{~nm}$ communication wavelength bands. The buried tunnel junction approach with epitaxial InP-based mirrors, although quite successful at longer wavelengths, suffers from reduced mirror contrast, and therefore, reduced RTCW single-mode powers at $1310 \mathrm{~nm}$ [5]. Additionally, epitaxial InP-based mirrors suffer from significantly reduced thermal conductivity compared to GaAs-AlGaAs. Similarly, GaInNAs-GaAs gain regions suffer sharp degradation of optical quality when moving from 1260 to $>1310 \mathrm{~nm}$, and operation at $1550 \mathrm{~nm}$ provides severe technological challenges. For these reasons, wafer bonding remains an attractive solution for long-wavelength VCSELs, because the thermally optimum mirror system (GaAs-AlGaAs) can be combined with the highest gain active material system (AlInGaAs-InP) to create maximum performance across the entire 1250-1650 nm range. This also makes this technology well suited for CWDM applications.

Early work on electrically pumped wafer-bonded VCSELs suffered from p-type free-carrier losses and large voltage drops at the p-p bonded interface [9]. The work of [2] and [3] circumvented this problem by introducing a vertically integrated 850-nm optical pump and a completely undoped 1310-nm VCSEL. The current work solves the problem by electrically bypassing the bonded interface using two intracavity contacts, and minimizing p-type absorption through the use of a buried tunnel junction. This provides a solution with room temperature single-mode performance comparable to [2], [3], but with a considerably simpler epitaxial structure and superior high-temperature performance.

\section{Device Structure}

Fig. 1 illustrates the present device structure. The cavity region consists of a p-i-n five quantum-well AlInGaAs active region with $1.1 \%$ compressive strain and strain compensated barriers. On top of this active region is a $\mathrm{p}+\mathrm{AlInAs}-\mathrm{n}+\mathrm{InP}$ patterned tunnel junction which is buried by a regrown $\mathrm{n}$-InP contact layer. The buried tunnel junction approach was pioneered by the authors of [4] and [5], in conjunction with epitaxially grown mirrors.

The etched tunnel junction introduces a step of about $300 \AA$ in the top surface of the regrown contact layer in Fig. 1. This surface is sufficiently planar to permit wafer bonding of an undoped 27- or 25-period 92\% AlGaAs-GaAs top mirror. The back mirror is also a wafer-bonded $92 \%$ AlGaAs-GaAs stack, 


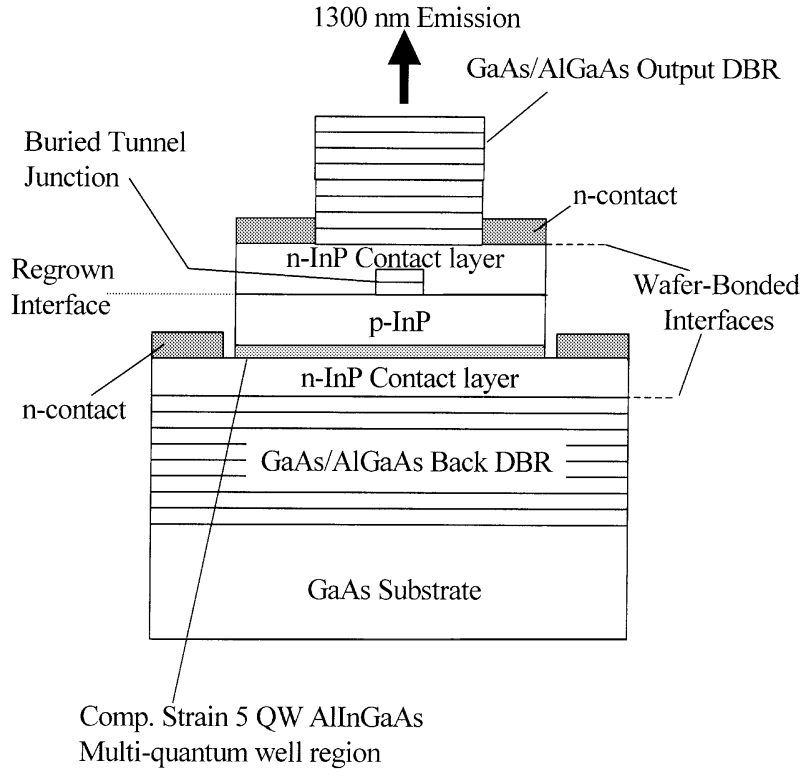

Fig. 1. Device structure of this work, employing two wafer-bonded AlGaAs distributed Bragg reflector mirrors and a buried tunnel junction.

consisting of 31 or 35 periods. Intracavity contacts are made to both top and bottom n-InP contact layers.

\section{DEVICE RESUlTS AND DisCusSiON}

Light-current-voltage temperature (LIVT) and spectral properties of devices with two different gain offsets were investigated. Fig. 2 illustrates the LIVT curve of an 8 - $\mu \mathrm{m}$ device operating at $1335 \mathrm{~nm}$. This device employs 27 periods on the output mirror, and 31 periods on the back mirror. Since the photoluminescence (PL) wavelength of the active material was at $1275 \mathrm{~nm}$, this device operated with a large PL offset of $60 \mathrm{~nm}$. The net result is a record maximum $\mathrm{CW}$ operating stage temperature of $134{ }^{\circ} \mathrm{C}$. Care was taken during temperature measurements to calibrate the measuring thermocouple. Fig. 3 illustrates the accompanying threshold versus temperature characteristic, illustrating a minimum $1-\mathrm{mA}$ threshold current at $90{ }^{\circ} \mathrm{C}$. These devices exhibit multimode behavior.

Fig. 4 illustrates the light-current temperature (LIT) curve of another $8-\mu \mathrm{m}$ device operating at $1320 \mathrm{~nm}$, with a smaller PL offset of approximately $45 \mathrm{~nm}$. This device employs 25 mirror periods on the output mirror and 35 on the back mirror. The device of Fig. 4 exhibits approximately 2-mW RTCW single-mode output power, and 1.0-mW RTCW single-mode power at $80{ }^{\circ} \mathrm{C}$. The maximum $\mathrm{CW}$ operating temperature of this device is $>110{ }^{\circ} \mathrm{C}$. These single-mode power results are close to the record results of [1], and at wavelengths $(1310-1320 \mathrm{~nm})$ where the GaInNAs-GaAs technology of [1] has not demonstrated good performance. Additionally, the single-mode power at elevated temperatures exceeds that of the integrated optically pumped approach [2], [3]. Fig. 5 illustrates the single-mode spectrum of this device at two temperatures, at currents near the rollover power.

The devices of Figs. 2-5 all exhibit a large series resistance of approximately $330 \Omega$. This arises both from a nonoptimized tunnel junction, and from the low $510^{17} \mathrm{~cm}^{-3}$ doping level of
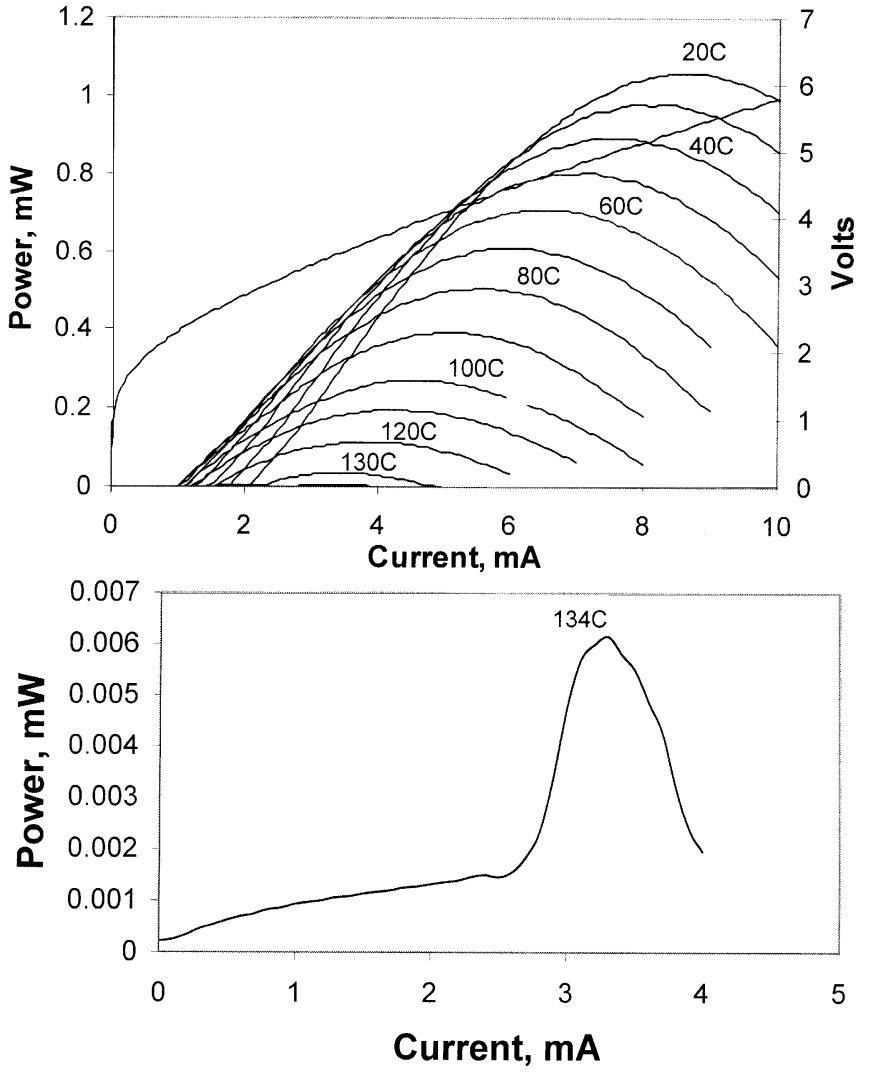

Fig. 2. The $8-\mu \mathrm{m}$ device with record $134{ }^{\circ} \mathrm{C}$ maximum $\mathrm{CW}$ operating temperature. Upper chart illustrates the $L-I-V$ curves from $20^{\circ}$ to $130^{\circ} \mathrm{C}$ (voltage is at $20^{\circ} \mathrm{C}$ ). Bottom chart shows the $L-I$ at the maximum $\mathrm{CW}$ lasing temperature of $134^{\circ} \mathrm{C}$.

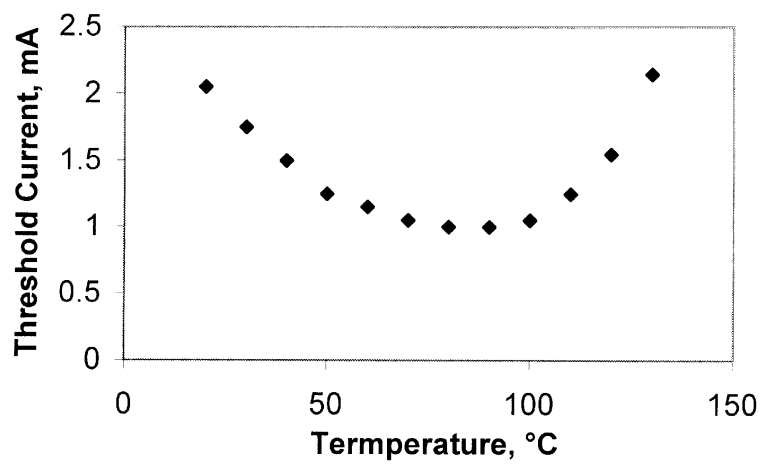

Fig. 3. Threshold current versus temperature for record high temperature device.

the n-type current spreading layers, in conjunction with the large diameter of both ring contacts injecting current into the buried tunnel junction aperture region. This large lateral resistance also leads to current crowding near the edges of the aperture. This in turn leads to the larger device diameters $(12 \mu \mathrm{m}$ and greater) exhibiting poor output power and temperature performance relative to the 8- $\mu \mathrm{m}$ apertures in Figs. 2-5.

\section{CONCLUSION}

The present work has demonstrated a device structure that overcomes the output power and temperature performance limitations of previous electrically pumped wafer-bonded 


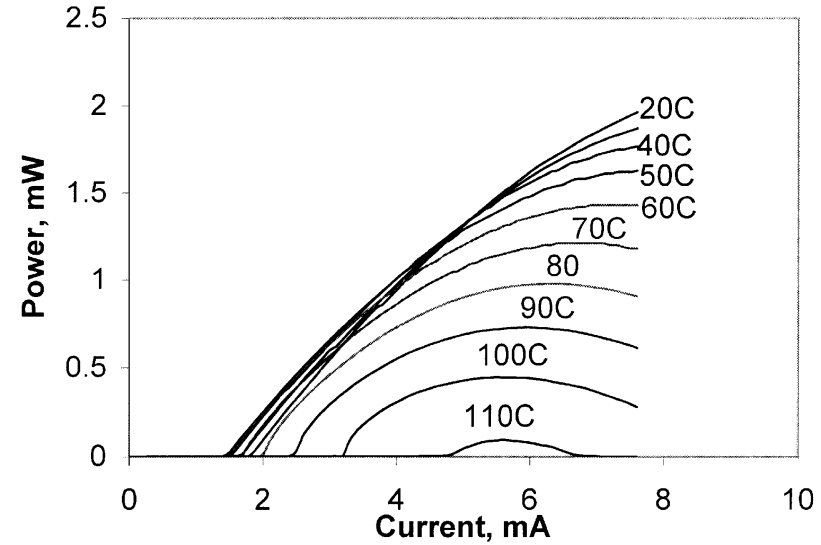

Fig. 4. $L-I$ curve of $8-\mu \mathrm{m}$ diameter single-mode device. The devices are single-mode up to the peak power shown in the graph at each temperature.

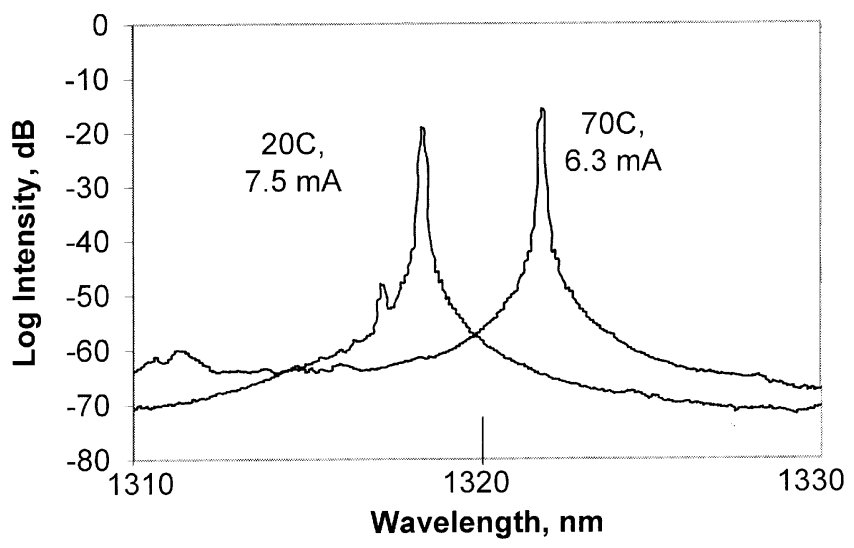

Fig. 5. Optical spectra near rollover at $20^{\circ}$ and $70^{\circ} \mathrm{C}$ for the device of Fig. 4. Wavelength range shown is from 1310 to $1330 \mathrm{~nm}$.

devices. The device structure has produced the highest published $\mathrm{CW}$ operating temperature $\left(134^{\circ} \mathrm{C}\right)$ of any VCSEL in the 1250-1650 $\mathrm{nm}$ range, and is the first VCSEL in the 1290-1360 $\mathrm{nm}$ band to demonstrate $>1-\mathrm{mW}$ CW single-mode power at $70{ }^{\circ} \mathrm{C}$.
The present devices still suffer from relatively large voltage due to a nonoptimized tunnel junction and excessive lateral current spreading resistance. Reduction of tunnel junction voltage has previously been demonstrated in [6], and theory indicates that considerable reduction of lateral spreading resistance can be accomplished by increasing $\mathrm{n}$-InP contact layer dopings to $10^{18} \mathrm{~cm}^{-3}$, resulting in negligible increase of free-carrier absorption, along with reduced diameter ring contacts. These improvements promise to further increase single-mode output power and temperature performance.

\section{REFERENCES}

[1] L. R. Thompson, L. M. F. Chirovsky, A. W. Jackson, R. L. Naone, D. Galt, S. R. Prakash, S. A. Feld, M. V. Crom, J. G. Wasserbauer, M. D. Lange, B. Mayer, and D. W. Kisker, "Performance of monolithic 1.3- $\mu \mathrm{m}$ VCSELs in telecom applications," presented at the Photonics West SPIE Conf., San Jose, CA, Jan. 2002, Paper OE 4649-05.

[2] V. Jayaraman, T. C. Goodnough, T. L. Beam, F. M. Ahedo, and R. A. Maurice, "Continuous-wave operation of single-transverse-mode VCSELs up to $115^{\circ} \mathrm{C}$," IEEE Photon. Technol. Lett., vol. 12, pp. 1595-1597, Dec. 2000.

[3] V. Jayaraman, "VCSEL makers pursue lower cost $1300 \mathrm{~nm}$ diodes," Laser Focus World, vol. 35, no. 8, pp. 159-168, Aug. 1999.

[4] R. Shau, M. Ortsiefer, J. Rosskopf, G. Bohm, F. kohler, and M. C. Amann, "Vertical cavity surface-emitting laser diodes at $1.55 \mu \mathrm{m}$ with large output power and high operation temperature," Electron. Lett., vol. 37 , no. 21 , pp. 1295-1296, Oct. 2001.

[5] G. Bohm, M. Ortsiefer, R. Shau, J. Rosskopf, C. Lauer, M. Maute, F. Kohler, F. Mederer, R. Meyer, and M. C. Amann, "InP-based VCSEL technology covering the range from 1.3 to $2.0 \mu \mathrm{m}$," J. Cryst. Growth, vol. 251, pp. 748-753, Apr. 2003.

[6] S. Nakagawa, E. Hall, G. Almuneau, J. K. Kim, D. A. Buell, H. Kroemer, and L. A. Coldren, " $88^{\circ} \mathrm{C}$ continuous wave operation of apertured intracavity contacted $1.55-\mu \mathrm{m}$ vertical cavity surface-emitting lasers," Appl. Phys. Lett., vol. 78, no. 10, pp. 1337-1339, Mar. 2001.

[7] N. Ledentsov, D. Bimberg, V. M. Ustinov, Z. I. Alferov, and J. A. Lott, "Quantum dots for VCSEL applications at $\lambda=1.3 \mu \mathrm{m}$," Physica E, vol. 13, pp. 871-875, Mar. 2002.

[8] N. Nishiyama, C. Caneau, G. Guryanov, X. S. Liu, M. Hu, and C. E. Zah, "High efficiency long-wavelength VCSEL on InP grown by MOCVD," Electron. Lett., vol. 39, no. 5, pp. 437-439, Mar. 2003.

[9] A. Karim, P. Abraham, D. Lofgreen, Y. J. Chiu, J. Piprek, and J. E. Bowers, "Wafer-bonded 1.55- $\mu \mathrm{m}$ vertical-cavity lasers with continuous-wave operation up to $105^{\circ} \mathrm{C}$," Appl. Phys. Lett., vol. 78, no. 18, pp. 2632-2633, Apr. 2001. 